\title{
Tin Oxynitride-based Ferroelectric Semiconductors for Solar Energy Conversion Applications
}

\author{
Steven T. Hartman, ${ }^{\dagger, \perp}$ Arashdeep S. Thind, ${ }^{\dagger}$ and Rohan Mishra ${ }^{\dagger, \dagger, *}$ \\ †Institute of Materials Science and Engineering, Washington University in St. Louis, One \\ Brookings Drive, St. Louis, MO 63130, USA \\ †Department of Mechanical Engineering and Materials Science, Washington University in St. \\ Louis, One Brookings Drive, St. Louis, MO 63130, USA \\ *Email: rmishra@,wustl.edu
}


ABSTRACT: Lead-halide perovskites have emerged as a promising class of semiconductors; however they suffer from issues related to lead-toxicity and instability. We report results of a firstprinciples-based design of heavy-metal-based oxynitrides as alternatives to lead-halide perovskites. We have used density-functional-theory calculations to search a vast composition space of $A B \mathrm{O}_{2} \mathrm{~N}$ and $A B \mathrm{ON}_{2}$ compounds, where $B$ is a $p$-block cation, and $A$ is an alkaline, alkaliearth, rare-earth or transition metal cation, and identify 10 new $A B \mathrm{O}_{2} \mathrm{~N}$ oxynitride semiconductors that we expect to be formable. Specifically, we discover a new family of ferroelectric semiconductors with $A^{3+} \mathrm{SnO}_{2} \mathrm{~N}$ stoichiometry $(A=\mathrm{Y}, \mathrm{Eu}, \mathrm{La}$, In, and $\mathrm{Sc})$ in the $\mathrm{LuMnO}_{3}$-type structure, which combine the strong bonding of metal oxides with the low carrier effective mass and small, tunable band gaps of the lead-halide perovskites. These tin oxynitrides have predicted direct band gaps ranging from $1.6-3.3 \mathrm{eV}$, and a sizeable electric polarization up to $17 \mu \mathrm{C} / \mathrm{cm}^{2}$, which is predicted to be switchable by an external electric field through a non-polar phase. With their unique combination of polarization, low carrier effective mass and band gaps spanning the entire visible spectrum, we expect $A \mathrm{SnO}_{2} \mathrm{~N}$ ferroelectric semiconductors will find useful applications as photovoltaics, photocatalysts, and for optoelectronics.

\section{TOC GRAPHICS}

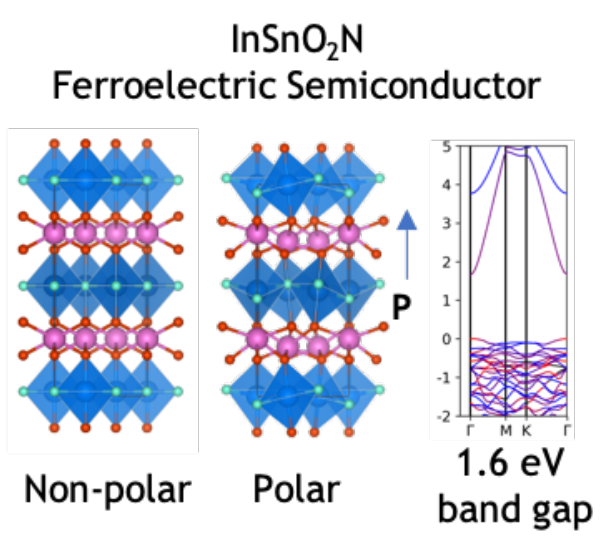


Lead-halide perovskites, such as methylammonium lead triiodide $\left(\mathrm{CH}_{3} \mathrm{NH}_{3} \mathrm{PbI}_{3}\right)$, have emerged as promising, high-performance semiconductors for optoelectronics and photovoltaics applications. Solar cells with lead-halide perovskite absorbers have surpassed power conversion efficiency of $25 \% .{ }^{1}$ These perovskites have tunable band gaps and high optical absorbance to efficiently utilize the incoming solar radiation. ${ }^{2}$ They possess long charge carrier lifetimes and large carrier diffusion lengths that enables effective separation of the photogenerated electrons and holes in solar cells. ${ }^{3-5}$ They exhibit high defect tolerance, ${ }^{6-9}$ wherein these semiconductors are able to retain the electronic properties of their pristine form even in the presence of common defects. ${ }^{9}$ ${ }^{10}$ There are also reports on the presence of ferroelectric order in some lead-halide perovskites. ${ }^{11-}$ ${ }^{14}$ In addition to the low effective mass of charge carriers in these perovskites, ${ }^{15}$ and their large dielectric constant ${ }^{16}$ another factor that can enhance carrier separation is the presence of ferroelectric domains wherein the in-built electric field can facilitate transport of the electrons and holes to opposite domain boundaries. ${ }^{17}$ Ordered domain structures can further provide pathways for efficient carrier extraction at the surface of the absorber layer. ${ }^{18}$ However, the extent to which ferroelectricity enhances charge separation in $\mathrm{CH}_{3} \mathrm{NH}_{3} \mathrm{PbI}_{3}$ is under significant debate as there are conflicting reports with some suggesting $\mathrm{CH}_{3} \mathrm{NH}_{3} \mathrm{PbI}_{3}$ to be ferroelectric ${ }^{11-14}$ while others implying it to be non-ferroelectric, ${ }^{19-21}$ or even antiferroelectric. ${ }^{22}$ Polar ordering in $\mathrm{CH}_{3} \mathrm{NH}_{3} \mathrm{PbI}_{3}$ is, at best, weak as it originates from the orientation of the rotating and vibrating polar organic cations $\left(\mathrm{CH}_{3} \mathrm{NH}_{3}{ }^{+}\right)$and their weak interaction with the neighboring halide ions through hydrogen bonds. ${ }^{18,}$ 20,23

Despite their superior performance, $\mathrm{CH}_{3} \mathrm{NH}_{3} \mathrm{PbI}_{3}$ and other organic lead-halide perovskites are plagued with environmental and thermodynamic instability, ${ }^{24,25}$ which combined with the highly toxic nature of lead, makes them less prospective for widespread commercialization. ${ }^{26,}{ }^{27}$ Hence, 
there is ongoing search for stable, lead-free perovskites that can emulate the performance of leadhalide perovskite-based semiconductors. ${ }^{3}{ }^{28-33}$ Such searches, for the most part, have been restricted to perovskite halides of heavier $p$-block cations such as $\mathrm{Ge}, \mathrm{Sn}$, and $\mathrm{Bi}$. The band edges of such compounds are made up of spatially delocalized $s$ or $p$ states which combine with the threedimensional corner connectivity of the perovskite framework to create dispersive bands with low carrier effective masses. ${ }^{15,34,35}$ However, these lead-free halides also suffer from long-term stability issues on exposure to ambient air, temperature and light. ${ }^{32}$ Their low stability can be attributed to the strength of the metal-halide bond, which is weaker than metal-oxide or -nitride bonds. ${ }^{36}$ In the context of stability, Bi-based oxide double perovskites, ${ }^{37}$ such as $\mathrm{KBaTeBiO}_{6},{ }^{33}$ have been recently shown as a promising composition space to search for lead-free replacements. However, none of the proposed alternatives have been able to replicate the increase in efficiency that lead-halide perovskite-based solar cells have undergone. The ideal replacement should display similar direct band gap, carrier effective masses, and absorbance as $\mathrm{CH}_{3} \mathrm{NH}_{3} \mathrm{PbI}_{3}$ with added stability. The performance of such semiconductors could be further enhanced if they display robust polarization.

In this work, we have used a first-principles computational approach to discover a new family of stable, Sn-based oxynitride ferroelectric semiconductors as potential replacements for leadhalide perovskites. Using density-functional-theory (DFT) calculations, we search a vast composition space of $A B \mathrm{O}_{2} \mathrm{~N}$ and $A B \mathrm{ON}_{2}$ stoichiometries, where $B$ is a $p$-block cation, and $A$ is an alkaline, alkali-earth, rare-earth or transition metal cation, and identify 10 new $A B \mathrm{O}_{2} \mathrm{~N}$ oxynitride compositions that we expect to be formable. Of these, the $A \mathrm{SnO}_{2} \mathrm{~N}$ family of five members ( $A=$ $\mathrm{Y}, \mathrm{Eu}, \mathrm{La}, \mathrm{In}$, and $\mathrm{Sc}$ ) stands out for its promising electronic properties. We find $\mathrm{YSnO}_{2} \mathrm{~N}$ and $\mathrm{EuSnO}_{2} \mathrm{~N}$ to be thermodynamically stable being on the convex hull of competing phases, and three 
others $A \mathrm{SnO}_{2} \mathrm{~N}$ ( $\left.A=\mathrm{La}, \mathrm{In}, \mathrm{Sc}\right)$ have a formation enthalpy $<94 \mathrm{meV} /$ atom from the hull, within the typical range of formability for oxides and nitrides. ${ }^{38}$ These Sn-oxynitrides adopt the $\mathrm{LuMnO}_{3}$ structure, ${ }^{39}$ in which a concerted motion of the $A$-site cations couples with polyhedral tilting ${ }^{40}$ to create a sizeable spontaneous polarization between $7-17 \mu \mathrm{C} / \mathrm{cm}^{2}$. We predict that these $A \mathrm{SnO}_{2} \mathrm{~N}$ materials to have band gaps ranging from 1.6 to $3.3 \mathrm{eV}$, with a dispersive conduction band leading to low electron effective masses of $(0.1-0.3) m_{0}$ (where $m_{0}$ is the mass of a free electron), values comparable to $\mathrm{CH}_{3} \mathrm{NH}_{3} \mathrm{PbI}_{3}{ }^{15}$ Specifically, we predict $\mathrm{InSnO}_{2} \mathrm{~N}$ - that has a direct band gap of $1.6 \mathrm{eV}$, a spontaneous polarization of $9.9 \mu \mathrm{C} / \mathrm{cm}^{2}$ and good formability $(50 \mathrm{meV} /$ atom above the convex hull) — to be a promising candidate for solar cell absorbers. With their unique combination of properties and band gaps spanning the entire visible spectrum, we expect $A \mathrm{SnO}_{2} \mathrm{~N}$ ferroelectric semiconductors will find useful applications as photovoltaics, photocatalysts, and for optoelectronics.

Our interest in oxynitrides stems from their potential to combine many of the favorable aspects of both halides and oxides. The poor stability of the heavy-metal halide perovskites could be overcome by replacing the halogen with oxygen or nitrogen, since metal oxides and nitrides are much more strongly bonded. However, the large electronegativity of oxygen results in a low valence band maximum (VBM), ${ }^{41}$ and the resulting materials have large band gaps, making them sub-optimal for solar energy harvesting. This band gap increase can potentially be mitigated by incorporating nitrogen to raise the VBM. ${ }^{42,43}$ For instance, while the oxide perovskite $\mathrm{CaNbO}_{3}$ shows a large band gap of $4.03 \mathrm{eV},{ }^{44}$ its isostructural oxynitride counterpart, $\mathrm{CaNbO}_{2} \mathrm{~N}$, shows a band gap of $2.1 \mathrm{eV} \cdot{ }^{43}$ Previous works on $A B \mathrm{O}_{2} \mathrm{~N}$ oxynitrides have mainly focused on transitionmetal cations at the $B$-site, especially those that prefer oxidation states of +4 and higher such as $\mathrm{Ta}^{5+}, \mathrm{Nb}^{5+}$, and $\mathrm{Hf}^{4+} .45,46$ These transition metal oxynitrides have proven useful for photocatalysis, 
but their localized $d$-states at the band edge reduce their electron and hole mobility. Because we want to find good semiconducting materials with dispersive bands and band gap in the visible region, we move beyond the known oxynitrides to focus on $p$-block cations at the $B$-site.

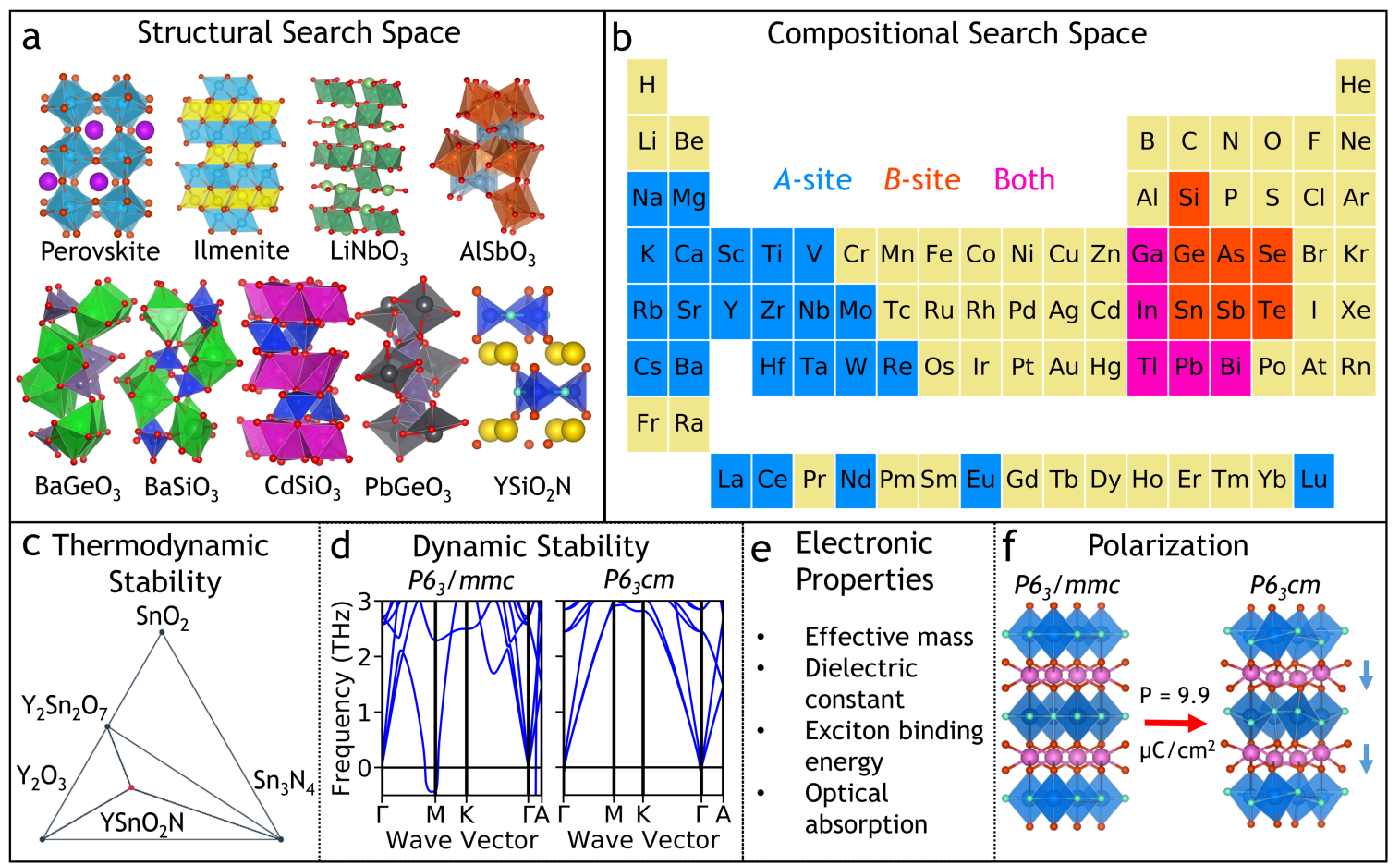

Figure 1. A schematic of the search strategy employed in this work to discover oxynitride semiconductors. a. We substituted common oxide and oxynitride structural prototypes with the $A$ - and $B$-site cations shown on the Periodic Table in b. c. All new materials were checked for thermodynamic stability or metastability versus known phases in the same composition space. d. We used mode analysis and phonon calculations to demonstrate the potential ferroelectricity of the stable $A \mathrm{SnO}_{2} \mathrm{~N}$ family of compounds. Finally, we calculated their e. electronic properties and f. spontaneous polarization.

Our search strategy for finding heavy-metal oxynitrides is illustrated in Figure 1 (see methods section and Supporting Information $\mathrm{S} 1$ for additional details). We choose $A$-site cations from the left side of the Periodic Table, while $B$ is a $p$-block cation, and we require that each composition be charge-balanced in common oxidation states of the cations. The charge-balance requirement 
restricts the number of $A B \mathrm{O}_{2} \mathrm{~N}$ and $A B \mathrm{ON}_{2}$ compositions to 130 and 76 , respectively. We then construct each composition in different structural prototypes observed in $\mathrm{ABO}_{3}$ oxides, including the perovskite-, $\mathrm{LiNbO}_{3^{-}}, \mathrm{FeTiO}_{3^{-}}, \mathrm{BaSiO}_{3^{-}}, \mathrm{CdSiO}_{3^{-}}, \mathrm{AlSbO}_{3^{-}}, \mathrm{GePbO}_{3^{-}}, \mathrm{BaGeO}_{3^{-}}$, and $\mathrm{YSiO}_{2} \mathrm{~N}$-structure types, as shown in Figure 1a. Then we calculate the new oxynitride's thermodynamic stability after structural relaxation. We do this by calculating the formation enthalpy of the most stable $A B \mathrm{O}_{2} \mathrm{~N}$ structure with respect to the convex hull of most stable reactants, which can be either elements or compounds, as found in the Materials Project database. ${ }^{47}$

This first step reveals that all the calculated $A B \mathrm{ON}_{2}$ materials are $>140 \mathrm{meV} /$ atom above the convex hull. While, they are within the formability limits of $90 \%$ of known binary nitrides (195 meV/atom), they are above that of known binary oxides $(94 \mathrm{meV} / \mathrm{atom}),{ }^{38}$ so they are not considered further. We find that $A B \mathrm{O}_{2} \mathrm{~N}$ compounds, with $B$ chosen from the $p$-block elements, do not typically adopt the common perovskite structure. We tested perovskites having different octahedral tilt patterns and found them to be $>100 \mathrm{meV} /$ atom above the convex hull (See Section $\mathrm{S} 2$ in Supporting Information). Instead, we find that the smaller $B$-site cations, such as $\mathrm{Si}^{4+}$ (Shannon radii ${ }^{48}$ of $0.4 \AA$ VI-coordinate), $\mathrm{As}^{5+}(0.46 \AA)$, or $\mathrm{Ge}^{4+}(0.53 \AA)$, favor tetrahedral coordination such as in $\mathrm{YSiO}_{2} \mathrm{~N}$, while the larger $\mathrm{Sn}^{4+}$ cation $(0.69 \AA)$ is more stable in a 5coordinate trigonal bipyramid coordination. We find this coordination is stabilized in the oxynitrides by anion ordering due to the higher site potential of the equatorial site, which we discuss further in Supporting Information Section S4. ${ }^{49-52}$ 


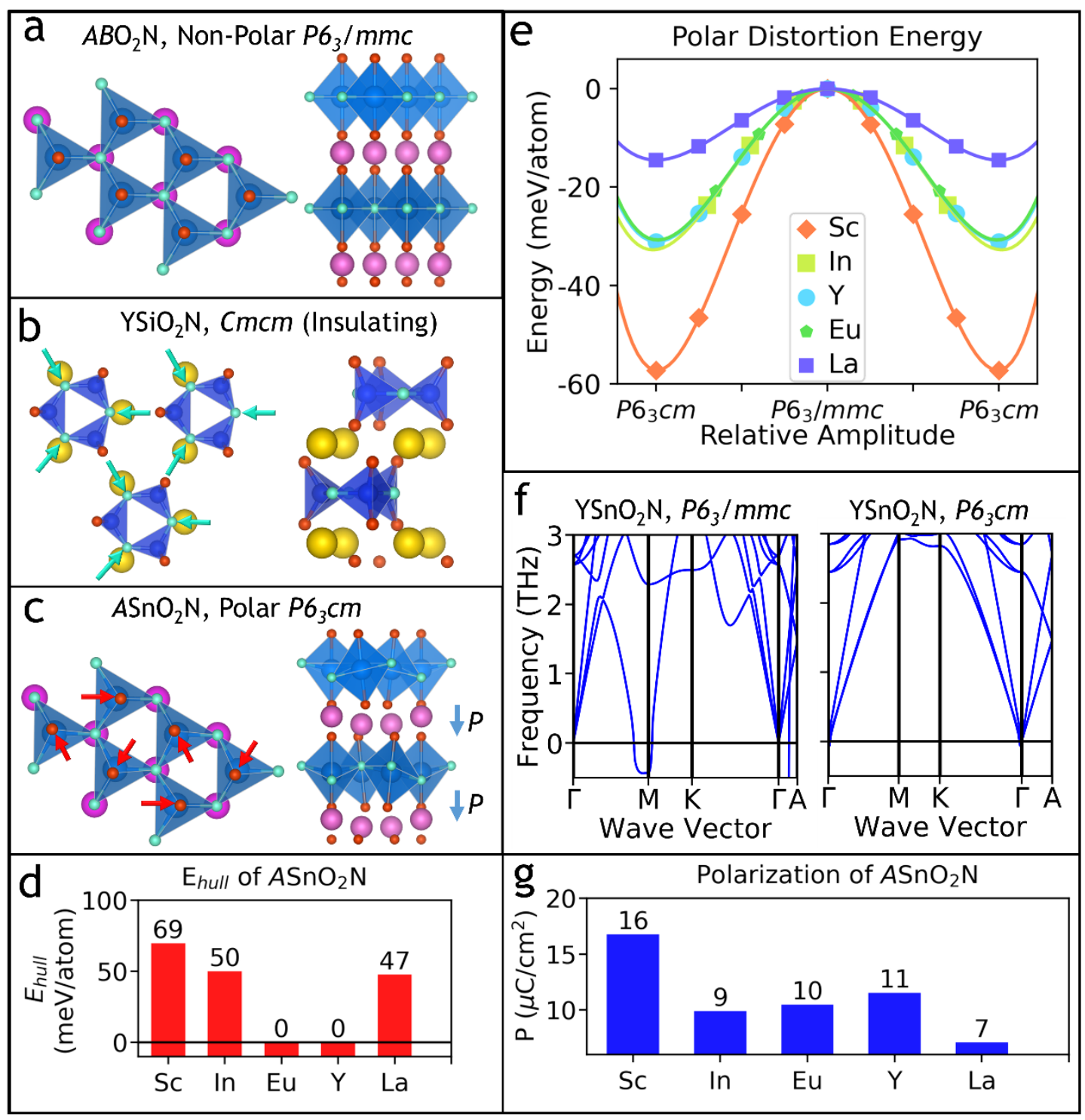

Figure 2. Structure, stability and polarization of $\boldsymbol{A B O}_{2} \mathbf{N}$. a. The centrosymmetric $P 6_{3} / m m c$ structure of $A B \mathrm{O}_{2} \mathrm{~N}$, with no polyhedral tilting. b. The polar and insulating $\mathrm{Cmcm}$ structure adopted by $\mathrm{YSiO}_{2} \mathrm{~N},{ }^{52}$ and other $A B \mathrm{O}_{2} \mathrm{~N}$ with small $B$ cations. The arrows illustrate the creation of isolated rings as the $B$-site coordination contracts. c. The polar $P 6_{3} \mathrm{~cm}$ structure, which is the ground state of $A \mathrm{SnO}_{2} \mathrm{~N}$ ( $\left.A=\mathrm{Sc}, \mathrm{In}, \mathrm{Eu}, \mathrm{Y}, \mathrm{La}\right)$. The arrows illustrate the polyhedral tilting mode that creates a polarization along the $c$-axis. d. Convex-hull energies of $A \mathrm{SnO}_{2} \mathrm{~N}$ with $A=\mathrm{Sc}$, In, $\mathrm{Eu}, \mathrm{Y}$, and La plotted in order of increasing Shannon ionic radius. e. The ferroelectric doublewell potential for $A \mathrm{SnO}_{2} \mathrm{~N}$ as they are switched through the centrosymmetric $P 6_{3} / m m c$ phase. f. The calculated phonon spectrum for $\mathrm{YSnO}_{2} \mathrm{~N}$, in the unstable centrosymmetric $P 6_{3} / m m c$ phase (left) with negative phonon frequencies, and the stable polar $\mathrm{P}_{3} \mathrm{~cm}$ phase (right) with strictly positive phonon frequencies. g. The calculated spontaneous polarization of $A \mathrm{SnO}_{2} \mathrm{~N}$ along the $c$-axis. 
While there are many possible structures with 4- or 5-coordination of the cations, the existence of anion-ordered $\mathrm{YSiO}_{2} \mathrm{~N}$ makes a group of three related structure types especially likely to be stable for $A B \mathrm{O}_{2} \mathrm{~N}$ compounds investigated here. ${ }^{52}$ These structures, and their symmetry relations, are illustrated in Figure 2. The high-symmetry 5-coordinate $P 6_{3} / m m c$ structure in Figure 2a has a two-dimensional network of corner-sharing trigonal bipyramids, and is known in a few materials such as high-pressure $\mathrm{InGaO}_{3} .{ }^{53}$ None of the $A B \mathrm{O}_{2} \mathrm{~N}$ compositions we have tested have a $P 6_{3} / m m c$ ground state, however. We find that reducing the size of the $B$-site cation contracts the coordination environment to tetrahedral, breaking the bonding network into the isolated rings as shown in Figure $2 \mathrm{~b}$, and observed in $\mathrm{YSiO}_{2} \mathrm{~N} .^{52}$ The $\mathrm{YSiO}_{2} \mathrm{~N}$-type structure has been represented with the 30 -atom centrosymmetric $\mathrm{Cmcm}$ unit cell in Figure 2a. While stacking patterns along the $c$-axis can reduce symmetry to polar $P 6122,54$ both the ring structures have wide band gaps and flat bands, which is a consequence of the breaking in polyhedral connectivity. ${ }^{15,34}$ We find that all $A B \mathrm{O}_{2} \mathrm{~N}$ compounds with $B=\mathrm{Si}, \mathrm{Ge}$, and As adopt this structure type, with $\mathrm{YSiO}_{2} \mathrm{~N}, \mathrm{EuGeO}_{2} \mathrm{~N}, \mathrm{LaGeO}_{2} \mathrm{~N}, \mathrm{BaAsO}_{2} \mathrm{~N}$, $\mathrm{CaAsO}_{2} \mathrm{~N}$, and $\mathrm{SrAsO}_{2} \mathrm{~N}$ having a formation enthalpy within $(0-94) \mathrm{meV} /$ atom above the convex hull, and are expected to be formable according to the metastability criterion defined above. As these $\mathrm{YSiO}_{2} \mathrm{~N}$-type materials are found to be wide-band-gap insulators with $\mathrm{PBE}$ band gaps $>2.5$ $\mathrm{eV}$ and have flat bands (see Supporting Information S5 for the example band structure of $\mathrm{YGeO}_{2} \mathrm{~N}$ ), we do not investigate them further.

Keeping the $B$-site fixed to $\mathrm{Sn}^{4+}$, but reducing the $A$-site radius, introduces polyhedral tilting to reduce the coordination number of $A$ from 8 to 7 , as observed in hexagonal manganite multiferroics. ${ }^{40}$ The tilting of the trigonal bipyramids, as shown in Figure 2c, removes the center of inversion symmetry located at the $A$-site, lowering the symmetry to polar $P 6{ }_{3} \mathrm{~cm},{ }^{40}$ and the $A$ - 
site cations displace along the $c$-axis. The unit cell triples to include six $A$ cations, of which four displace along one direction, and two along the opposite direction, creating a sizeable polarization of $\sim 10 \mu \mathrm{C} / \mathrm{cm}^{2}$. This transition, which is driven by the size mismatch of the two cations, is known as a geometric ferroelectric transition, in contrast to ferroelectrics driven by lone-pair stereochemistry, such as $\mathrm{BiFeO}_{3}$, or by second-order Jahn-Teller distortions observed in compounds with $d^{0}$ cations, such as $\mathrm{BaTiO}_{3}{ }^{55-57}$ We find two compounds with the $P 6_{3} m c$ structure, $\mathrm{EuSnO}_{2} \mathrm{~N}$ and $\mathrm{YSnO}_{2} \mathrm{~N}$, are on the convex hull and are expected to be stable against decomposition. We also find $\mathrm{InSnO}_{2} \mathrm{~N}, \mathrm{LaSnO}_{2} \mathrm{~N}$, and $\mathrm{ScSnO}_{2} \mathrm{~N}$ are within $94 \mathrm{meV} /$ atom of the hull, as shown in Figure 2d, and we expect them to be experimentally formable. Creation of a single $\mathrm{O}^{2-} / \mathrm{N}^{3-}$ antisite pair in $\mathrm{InSnO}_{2} \mathrm{~N}$ costs $1.14 \mathrm{eV}$, indicating a strict anion ordering, which contrasts with the partial order of the octahedral perovskite oxynitrides. ${ }^{58}$ Due to their stability, polarization, and beneficial electronic properties, these compounds are the most promising result of our search, and we focus on them for the remainder of this article.

Having identified a set of stable and polar tin oxynitrides, we now look at the ferroelectric transition in more detail. We plot the energy difference between the non-polar $P 6_{3} / m m c$ and polar $P 6_{3} m c$ structures in Figure 2e, because this value can be used to approximate the maximum size of the energy barrier for ferroelectric switching. For comparison, isostructural $\mathrm{YMnO}_{3}$ has a calculated barrier of $21 \mathrm{meV} / \mathrm{atom},{ }^{47}$ and an experimental ferroelectric transition temperature $T_{\mathrm{C}}$ $=914 \mathrm{~K} .{ }^{59}$ The comparable barrier size of $15-30 \mathrm{meV} /$ atom for $A=\mathrm{In}, \mathrm{Y}, \mathrm{Eu}, \mathrm{La}$ leads us to predict the polarization of these $A \mathrm{SnO}_{2} \mathrm{~N}$ to be robust and yet switchable. $\mathrm{ScSnO}_{2} \mathrm{~N}$, at $57 \mathrm{meV} /$ atom, may also be switchable, if the dielectric breakdown field is large. As an additional check, we have calculated the phonon modes of the $P 6_{3} / m m c$ and $P 6_{3} m c$ structures of $\mathrm{YSnO}_{2} \mathrm{~N}$; the negative frequencies of $P 6_{3} / m m c$ in Figure $2 \mathrm{f}$ indicate that the ferroelectric mode is soft, while the polar 
$P 6_{3} m c$ has no soft modes and is dynamically stable. Finally, we have calculated the polarization of the five stable or metastable tin oxynitrides, which ranges from 7.1 to $16.8 \mu \mathrm{C} / \mathrm{cm}^{2}$, and we show the results in Figure 2g. As polar materials, the $A \mathrm{SnO}_{2} \mathrm{~N}$ family also have relatively large static dielectric constants $\varepsilon \approx 17$, which is beneficial for screening charged defects. ${ }^{8}$

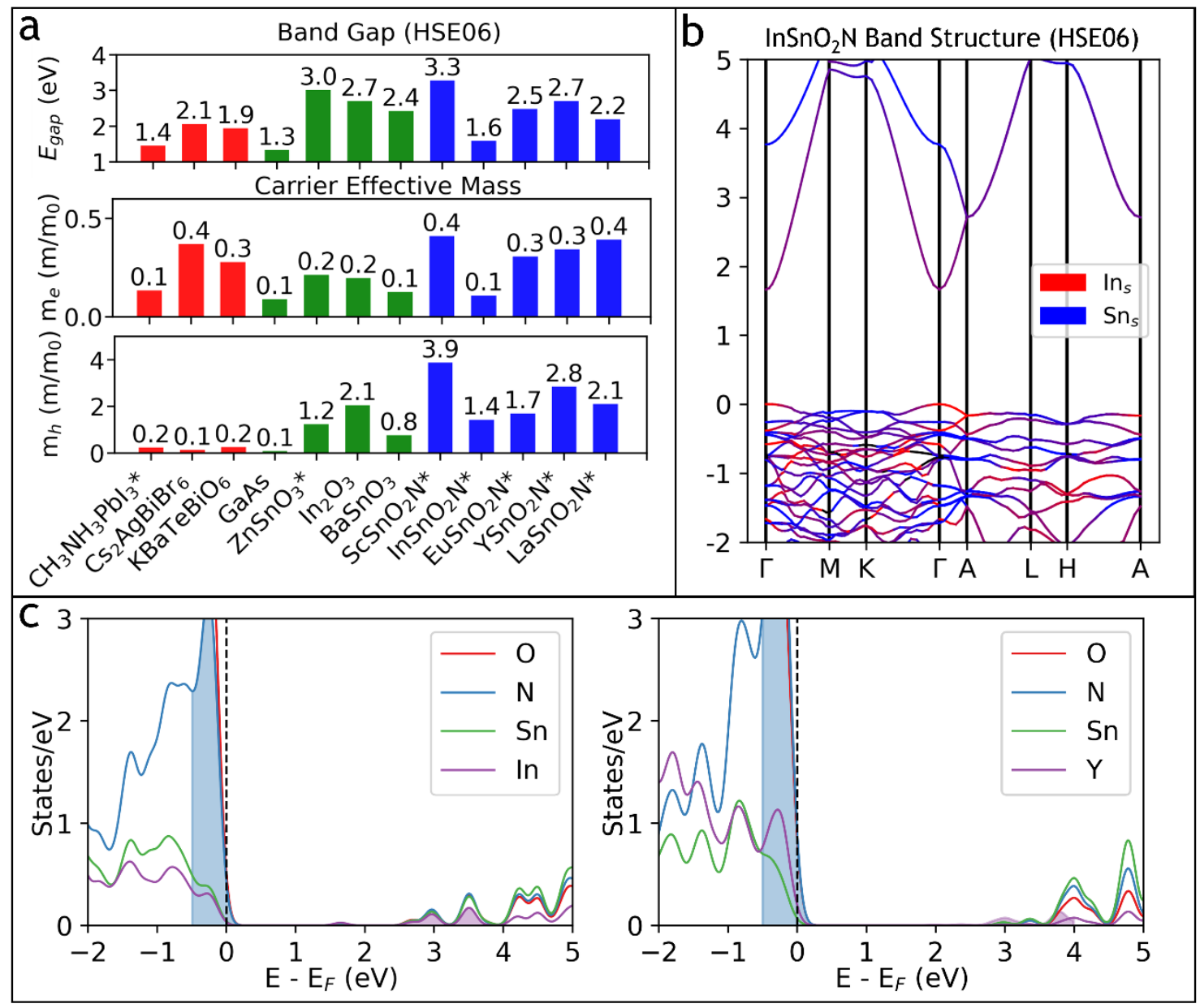

Figure 3. Electronic properties of $\mathbf{A S n O}_{2} \mathbf{N}$ compounds. a. Top: HSE06 band gap values for $A \mathrm{SnO}_{2} \mathrm{~N}$ (blue) compounds with $A=\mathrm{Sc}, \mathrm{In}, \mathrm{Eu}, \mathrm{Y}$, and $\mathrm{La}$, compared to literature values ${ }^{33,88-90}$ for several other $n$-type semiconductors (green) and perovskites (red). Middle: The effective electron masses for the same materials, calculated with the PBE functional. ${ }^{47}$ Bottom: the effective hole masses. Polar materials are marked with an asterisk *. b. The electronic band structure of $\mathrm{InSnO}_{2} \mathrm{~N}$, calculated using the HSE06 functional. The red and blue colors show the relative contributions of In $s$-states and Sn $s$-states, respectively. c. The HSE06 density of electronic states for $\mathrm{InSnO}_{2} \mathrm{~N}$ (left) and $\mathrm{YSnO}_{2} \mathrm{~N}$ (right), projected onto the orbitals of individual atoms. The VBM nitrogen states and CBM In/Y states are shaded. 
Since the $A \mathrm{SnO}_{2} \mathrm{~N}$ compounds are stable and polar, we calculate their band gaps with the more accurate HSE06 functional, ${ }^{60}$ to investigate their promise for solar energy harvesting applications. We find that changing the $A$-site also allows the band gap to be tuned from 1.6 to $3.3 \mathrm{eV}$, spanning the entire visible spectrum and approaching the gap of $\mathrm{CH}_{3} \mathrm{NH}_{3} \mathrm{PbI}_{3}$, which has a band gap of 1.55 $\mathrm{eV},{ }^{61}$ close to the ideal for photovoltaic absorbers. We show the calculated HSE06 band gaps of $A \mathrm{SnO}_{2} \mathrm{~N}$ in Figure 3a, compared with several other known perovskite photovoltaic absorbers and $n$-type semiconductors, which serve as benchmarks. Their band gaps also compare favorably for solar energy harvesting to Sn-based perovskites: $\mathrm{BaSnO}_{3}$ and $\mathrm{SrSnO}_{3}$, which have indirect theoretical (HSE06) band gaps of $2.43 \mathrm{eV}$ and $3.50 \mathrm{eV},{ }^{62}$ respectively, and experimentally observed gaps $>3 \mathrm{eV} .{ }^{63}$

We also calculate the full electronic band structures, using the PBE functional due to computational constraints. Projecting the electronic bands onto atomic orbitals reveals that the VBM of $A B \mathrm{O}_{2} \mathrm{~N}$ is composed of both $\mathrm{O}$ and $\mathrm{N}$ states, as expected. The CBM, meanwhile, is highly dispersive with contributions from the s-orbitals of all four atoms, indicating that the bonding is covalent, with electrons partially transferred from cation to anion.

$\mathrm{InSnO}_{2} \mathrm{~N}$ has a small band gap of $1.6 \mathrm{eV}$, compared to the $2.7 \mathrm{eV}$ gap of $\mathrm{YSnO}_{2} \mathrm{~N}$, and the effect cannot be attributed to polyhedral tilting reducing the degree of covalent orbital overlap. $\mathrm{Y}^{3+}$ cation is only $0.10 \AA$ larger than $\mathrm{In}^{3+}$, which leads to smaller tilting of the Sn-based octahedra in $\mathrm{YSnO}_{2} \mathrm{~N}$ compared to $\mathrm{InSnO}_{2} \mathrm{~N}$, and so by this metric alone $\mathrm{InSnO}_{2} \mathrm{~N}$ ought to have a larger gap. The small gap of $\mathrm{InSnO}_{2} \mathrm{~N}$ is instead caused by the high electronegativity of In (1.78) compared with $\mathrm{Y}$ (1.22) ${ }^{64}$ which increases the strength of the covalent In-O and In-N interactions. Specifically, the In $s$-orbitals lie only $2.15 \mathrm{eV}$ above those of $\mathrm{Sn}$, compared to $5.93 \mathrm{eV}$ for $\mathrm{Y},{ }^{65}$ indicating that the conduction band will have a substantial In $s$ character as well as $\operatorname{Sn} s$. Examination of the atom- 
projected density of states obtained with the HSE06 functional, as shown in Figure 3c, shows a large contribution of In s-states to the conduction band edge. We further examine the atomic contribution to the $\mathrm{CBM}$ in $\mathrm{YSnO}_{2} \mathrm{~N}$ and $\mathrm{InSnO}_{2} \mathrm{~N}$. Indium and tin atoms contribute $30 \%$ and $24 \%$, respectively, to the $\mathrm{CBM}$ (at $\Gamma$-point) in $\mathrm{InSnO}_{2} \mathrm{~N}$, whereas $\mathrm{Y}$ and $\mathrm{Sn}$ contribute $8 \%$ and $32 \%$, respectively, to the $\mathrm{CBM}$ in $\mathrm{YSnO}_{2} \mathrm{~N}$. So, we conclude that the contribution from the low-lying In states significantly lowers the $\mathrm{CBM}$ of $\mathrm{InSnO}_{2} \mathrm{~N}$. $\mathrm{InSnO}_{2} \mathrm{~N}$ also has a direct band gap, compared to the indirect gaps of the other four tin oxynitrides, which are $<0.2 \mathrm{eV}$ smaller than their direct gaps. We have confirmed the direct nature of the band gap by re-calculating $\mathrm{InSnO}_{2} \mathrm{~N}$ 's band structure using HSE06, which we show in Figure $3 b$.

Photovoltaic applications require good charge carrier mobility to reduce non-radiative recombination losses and efficiently transport the photo-generated carriers to the electrodes. To estimate the carrier mobility, we have calculated the effective mass of electrons and holes in $A \mathrm{SnO}_{2} \mathrm{~N}$ oxynitrides from their band structure obtained using PBE. The effective mass of their carriers are shown in Figure 3a along with that of other promising semiconductors, such as $\mathrm{CH}_{3} \mathrm{NH}_{3} \mathrm{PbI}_{3}$, lead-free double perovskites $\mathrm{Cs}_{2} \mathrm{AgBiO}_{6}$ and $\mathrm{KBaTeBiO}_{6},{ }^{33} \mathrm{GaAs}, \mathrm{In}_{2} \mathrm{O}_{3}$, and $\mathrm{Sn}-$ based semiconductors $\mathrm{BaSnO}_{3}$ and $\mathrm{ZnSnO}_{3} .{ }^{47,66}$ All five oxynitrides have very light electrons, with $\mathrm{InSnO}_{2} \mathrm{~N}$ having $m_{\mathrm{e}}=0.11 m_{0}$. This compares favorably to the calculated $m_{\mathrm{e}}$ of $\mathrm{CH}_{3} \mathrm{NH}_{3} \mathrm{PbI}_{3}$ $\left(0.14 m_{0}\right), \mathrm{Cs}_{2} \mathrm{AgBiO}_{6}\left(0.37 m_{0}\right)$, and $\mathrm{KBaTeBiO}_{6}\left(0.28 m_{0}\right)$. The holes are heavier as is typical for most metal oxides, with $\mathrm{InSnO}_{2} \mathrm{~N}$ having holes with an effective mass of $1.43 m_{0}$. Oxide and nitride semiconductors are inherently $n$-type doped due to native anion vacancies. Therefore, the extremely low effective mass of the electrons in $A \mathrm{SnO}_{2} \mathrm{~N}$ oxynitrides is expected to make them promising semiconductors. 
Furthermore, for solar cell applications, a smaller excitonic binding energy is desirable, such that the photogenerated electron-hole pairs can be separated without recombination. The light electrons and high dielectric constant suggest that excitons can be separated easily if $\mathrm{InSnO}_{2} \mathrm{~N}$ is used as a light absorber. We calculate the exciton binding energy $\left(E_{\text {bind }}\right)$ using the Wannier-Mott model ${ }^{67}$ :

$$
E_{\text {bind }}=\frac{m_{e}^{*} m_{h}^{*}}{\left(m_{e}^{*}+m_{h}^{*}\right) m_{0} \varepsilon_{\text {stat }}^{2}} \mathrm{R}_{\mathrm{H}}
$$

where $m_{e}{ }^{*}$ and $m_{h}{ }^{*}$ are the electron and hole effective masses, respectively, $\mathrm{R}_{\mathrm{H}}$ is the Rydberg constant $(13.6 \mathrm{eV}), m_{0}$ is the free electron mass, and $\varepsilon_{\text {stat }}$ is the static dielectric constant calculated to be 18 using PBE for $\mathrm{InSnO}_{2} \mathrm{~N}$. We obtain $E_{\text {bind }}=4.6 \mathrm{meV}$ from the out-of-plane effective masses and dielectric constant for $\mathrm{InSnO}_{2} \mathrm{~N}$ (using in-plane values changes $E_{\text {bind }}$ to $7.2 \mathrm{meV}$ ). This value is much less than the thermal energy $\sim 27 \mathrm{meV}$ at room temperature, so we predict that excitons will separate easily, which is another similarity between $\mathrm{InSnO}_{2} \mathrm{~N}$ and $\mathrm{MAPbI}_{3}$. While the calculated and measured values of $E_{\text {bind }}$ for $\mathrm{MAPbI}_{3}$ span two orders of magnitude depending on the methods used, most of the reported values cluster in the region from 10 to $50 \mathrm{meV} .{ }^{68}$

In addition to the fast exciton separation, we expect the photovoltaic performance of $\mathrm{InSnO}_{2} \mathrm{~N}$ based solar cells to be further enhanced by the bulk photovoltaic effect (BPVE) ${ }^{69}$ wherein its lack of inversion symmetry and sizeable spontaneous polarization can generate a large photovoltage. BPVE is dominated by the shift current mechanism, in which the asymmetric potential in polar materials leads to continuous excitation of electrons and holes under illumination to quasiparticle coherent states having intrinsic momenta, that in turn result in a large photocurrent. ${ }^{70,71}$ The shift current can be further enhanced by controlling the geometry of domains and domain walls in ferroelectrics. ${ }^{72,} 73$ The formation of Schottky barriers at the ferroelectric absorber/electrode interfaces, ${ }^{74}$ can also improve the photovoltaic efficiency. However, existing ferroelectric 
materials based on transition metal compounds, such as $\mathrm{PbTiO}_{3}$ and $\mathrm{BiFeO}_{3}$ have wide band gaps, and cannot efficiently utilize the solar spectrum. To increase the absorbance and enhance BPVE of ferroelectrics, numerous attempts have been made to reduce their band gap. Alloying different transition metals and controlling their topological order, such as in $\mathrm{BiFe}_{1-x} \mathrm{Co}_{x} \mathrm{O}_{6},\left[\mathrm{KNbO}_{3}\right]_{1-x}$ $\left[\mathrm{BaNi}_{0.5} \mathrm{Nb}_{0.5} \mathrm{O}_{3}\right]$, and $\mathrm{BiFeCrO}_{6}$ is a popular method, and can lead to ferroelectric oxides with band gap as small as $1.4 \mathrm{eV} ; ;^{75-77}$ however, these strategies have led to only small improvements in photoconversion efficiency. First-principles and model calculations of the shift current have attributed this modest improvements of BPVE in transition-metal-based ferroelectrics to the localized nature of their band edges that are comprised of $d$-states. ${ }^{70}$ It has been predicted that BPVE can be significantly improved by using ferroelectric materials having delocalized $s$ or $p$ states at the band edges and an optimal band gap $\sim 1.6 \mathrm{eV}$ to maximize the absorption of the solar spectrum. ${ }^{70}$ Based on these, we predict the new tin oxynitrides reported here could be well-suited to harness BPVE and lead to high photoconversion efficiencies.

In summary, our first-principles search has revealed the stability of a family of $A \mathrm{SnO}_{2} \mathrm{~N}$ compounds which adopt the polar $\mathrm{LuMnO}_{3}$ structure. By incorporating the $p$-block element $\mathrm{Sn}$ into a quaternary oxynitride, we obtain good electronic properties with a highly dispersive conduction band and mobile conduction electrons, without sacrificing stability. We further predict that the band gap in these new tin oxynitrides can be tuned across the entire visible spectrum by varying the $A$-site cation. The combination of delocalized $s$-states at the conduction band edge, polar symmetry and band gap tunability, makes them attractive platforms to harness BPVE and make high-efficiency solar cells with photovoltages beyond the band gap. Furthermore, the presence of delocalized states at the band edge and the large dielectric constant, could potentially makes these semiconductors defect tolerant. Our initial results of antisite disorder between In and 
$\mathrm{Sn}$ in $\mathrm{InSnO}_{2} \mathrm{~N}$ shows that while these defect pairs can form easily - having a formation energy of $0.20 \mathrm{eV}$ - they don't result in any mid-gap states that can act as non-radiative recombination centers. The ability to substitute cations at the $A$-site and control the stoichiometry of the $\mathrm{O} / \mathrm{N}$ anions could enable ambipolar doping for various applications. Overall, we expect these $\mathrm{ASnO}_{2} \mathrm{~N}$ oxynitrides will enable several promising applications in solar energy harvesting through efficient solar cells and photocatalysts, and for visible-light optoelectronics.

\section{COMPUTATIONAL METHODS}

Materials screening: We have calculated the energy and electronic ground state of $206 A B \mathrm{O}_{3-x} \mathrm{~N}_{x}$ compositions in approximately 1,500 total relaxed structures, using DFT calculations as implemented in the Vienna Ab initio Simulation Package (VASP) 78,79 according to the parameters of the Materials Project. ${ }^{47}$ This involves the use of the PBE functional ${ }^{80}$ with a plane-wave cutoff of $520 \mathrm{eV}$ and a $k$-point density of 1000 per reciprocal atom. Most calculations used the tetrahedron method of Brillouin zone integration, ${ }^{81}$ but a few could not be converged this way and were recalculated with a Gaussian smearing of $<0.1 \mathrm{eV}$. All of these calculations were set up in an initial ferromagnetic configuration, but the magnetic moments were free to relax. Calculations were set up and run using the Pymatgen and Atomate libraries, ${ }^{82,83}$ and the convex hull energies were calculated relative to the other phases in the Materials Project database. We provide in Supporting Information S1 a detailed discussion of which cation compositions and structural polymorphs we searched in this study.

Dielectric and piezoelectric properties: We calculated the dielectric and piezoelectric tensors, including both electronic and ionic contributions, using density functional perturbation theory ${ }^{84}$ $($ LEPSILON $=$ TRUE, LPEAD $=$ TRUE $)$ and a $k$-points grid of $9 \times 5 \times 5$ points. To increase 
accuracy, we turned off real-space evaluation of projection operators (LREAL $=$ FALSE) and increased the plane-wave energy cutoff to $600 \mathrm{eV}$. Spontaneous polarization was calculated according to the Berry phase method, ${ }^{85}$ with the polarization branch between $P 6_{3} / \mathrm{mmc}$ and $\mathrm{P}_{3} \mathrm{~cm}$ fit using three intermediate images interpolated with ISODISTORT. ${ }^{86}$ Phonon spectra were calculated using the Pymatgen interface to the Phonopy software. ${ }^{87}$

Electronic and optical properties: We tested the effect of spin-orbit coupling for heavy elements such as La and Sn, but found that it was not required for an accurate band structure (see Supporting Information S6). We calculated the HSE06 band gaps using a plane wave cutoff of $500 \mathrm{eV}$ and a $4 \times 4 \times 2 \Gamma$-centered $k$-points grid, downsampled by a factor of two for the exact exchange potential $($ NKRED $=2)$.

\section{ASSOCIATED CONTENT}

Supporting Information: The Supporting Information is available free of charge at DOI:

Structural polymorphs used for screening $A B \mathrm{O}_{2} \mathrm{~N}$ and $A B \mathrm{ON}_{2}$ compounds; $A \mathrm{SnO}_{2} \mathrm{~N}$ perovskites with different anion-ordering and octahedral tilting; $\mathrm{ASbO} 2 \mathrm{~N}$ structure; site potentials in $\mathrm{InSnO}_{2} \mathrm{~N}$; electronic band structure of $A B \mathrm{O}_{2} \mathrm{~N}$ with rings of $\mathrm{B}$-centered tetrahedra; effect of spin-orbit coupling; cation-site disorder in $\mathrm{InSnO}_{2} \mathrm{~N}$.

\section{AUTHOR INFORMATION}

Corresponding author: *Email: rmishra@wustl.edu 
Present Address: ${ }^{\perp}$ (S.T.H.) Materials Science and Technology Division, Los Alamos National Laboratory, Los Alamos, NM 87545, USA.

Conflicts of interest: The authors declare no competing financial interest.

\section{ACKNOWLEDGMENTS}

This work was supported by the National Science Foundation (NSF) grant number 1806147. This work used computational resources of the Extreme Science and Engineering Discovery Environment (XSEDE), which is supported by NSF grant number ACI-1548562. 


\section{REFERENCES}

1. NREL Best Research-Cell Efficiency Chart. Available at https://www.nrel.gov/pv/cellefficiency.html (Accessed on February 4, 2020).

2. Stoumpos, C. C.; Kanatzidis, M. G., Halide Perovskites: Poor Man's High-Performance Semiconductors. Adv Mater 2016, 28 (28), 5778-93.

3. Stoumpos, C. C.; Malliakas, C. D.; Kanatzidis, M. G., Semiconducting Tin and Lead Iodide Perovskites with Organic Cations: Phase Transitions, High Mobilities, and Near-Infrared Photoluminescent Properties. Inorganic Chemistry 2013, 52 (15), 9019-9038.

4. Dong, Q.; Fang, Y.; Shao, Y.; Mulligan, P.; Qiu, J.; Cao, L.; Huang, J., Solar cells. Electron-hole diffusion lengths $>175$ mum in solution-grown $\mathrm{CH} 3 \mathrm{NH} 3 \mathrm{PbI} 3$ single crystals. Science 2015, 347 (6225), 967-70.

5. Saidaminov, M. I.; Abdelhady, A. L.; Murali, B.; Alarousu, E.; Burlakov, V. M.; Peng, W.; Dursun, I.; Wang, L.; He, Y.; Maculan, G.; Goriely, A.; Wu, T.; Mohammed, O. F.; Bakr, O. M., High-quality bulk hybrid perovskite single crystals within minutes by inverse temperature crystallization. Nat Commun 2015, 6, 7586.

6. Yin, W. J.; Shi, T.; Yan, Y., Unique properties of halide perovskites as possible origins of the superior solar cell performance. Adv Mater 2014, 26 (27), 4653-8.

7. Yin, W.-J.; Shi, T.; Yan, Y., Unusual defect physics in CH3NH3PbI3 perovskite solar cell absorber. Applied Physics Letters 2014, 104 (6), 063903.

8. Du, M. H., Efficient carrier transport in halide perovskites: theoretical perspectives. $J$. Mater. Chem. A 2014, 2 (24), 9091-9098. 
9. Thind, A. S.; Luo, G.; Hachtel, J. A.; Morrell, M. V.; Cho, S. B.; Borisevich, A. Y.; Idrobo, J. C.; Xing, Y.; Mishra, R., Atomic Structure and Electrical Activity of Grain Boundaries and Ruddlesden-Popper Faults in Cesium Lead Bromide Perovskite. Adv Mater 2019, 31 (4), 1805047.

10. Morrell, M. V.; He, X. Q.; Luo, G. F.; Thind, A. S.; White, T. A.; Hachtel, J. A.; Borisevich, A. Y.; Idrobo, J. C.; Mishra, R.; Xing, Y. C., Significantly Enhanced Emission Stability of $\mathrm{CsPbBr} 3$ Nanocrystals via Chemically Induced Fusion Growth for Optoelectronic Devices. Acs Applied Nano Materials 2018, 1 (11), 6091-6098.

11. Röhm, H.; Leonhard, T.; Hoffmann, M. J.; Colsmann, A., Ferroelectric domains in methylammonium lead iodide perovskite thin-films. Energy \& Environmental Science 2017, 10 (4), 950-955.

12. Kutes, Y.; Ye, L.; Zhou, Y.; Pang, S.; Huey, B. D.; Padture, N. P., Direct Observation of Ferroelectric Domains in Solution-Processed CH3NH3PbI3 Perovskite Thin Films. $J$ Phys Chem Lett 2014, 5 (19), 3335-9.

13. Rakita, Y.; Bar-Elli, O.; Meirzadeh, E.; Kaslasi, H.; Peleg, Y.; Hodes, G.; Lubomirsky, I.; Oron, D.; Ehre, D.; Cahen, D., Tetragonal CH3NH3PbI3 is ferroelectric. Proc Natl Acad Sci US A 2017, 114 (28), E5504-E5512.

14. Garten, L. M.; Moore, D. T.; Nanayakkara, S. U.; Dwaraknath, S.; Schulz, P.; Wands, J.; Rockett, A.; Newell, B.; Persson, K. A.; Trolier-McKinstry, S.; Ginley, D. S., The existence and impact of persistent ferroelectric domains in MAPbI3. Sci Adv 2019, 5 (1), eaas9311. 
15. Thind, A. S.; Huang, X.; Sun, J. W.; Mishra, R., First-Principles Prediction of a Stable Hexagonal Phase of CH3NH3PbI3. Chemistry of Materials 2017, 29 (14), 6003-6011.

16. Lin, Q.; Armin, A.; Nagiri, R. C. R.; Burn, P. L.; Meredith, P., Electro-optics of perovskite solar cells. Nat Photon 2015, 9 (2), 106-112.

17. Frost, J. M.; Butler, K. T.; Brivio, F.; Hendon, C. H.; van Schilfgaarde, M.; Walsh, A., Atomistic origins of high-performance in hybrid halide perovskite solar cells. Nano Lett 2014, 14 (5), 2584-90.

18. Frost, J. M.; Butler, K. T.; Walsh, A., Molecular ferroelectric contributions to anomalous hysteresis in hybrid perovskite solar cells. APL Materials 2014, 2 (8), 081506.

19. Liu, Y.; Collins, L.; Proksch, R.; Kim, S.; Watson, B. R.; Doughty, B.; Calhoun, T. R.; Ahmadi, M.; Ievlev, A. V.; Jesse, S.; Retterer, S. T.; Belianinov, A.; Xiao, K.; Huang, J.; Sumpter, B. G.; Kalinin, S. V.; Hu, B.; Ovchinnikova, O. S., Chemical nature of ferroelastic twin domains in $\mathrm{CH} 3 \mathrm{NH} 3 \mathrm{PbI} 3$ perovskite. Nat Mater 2018, 17 (11), 1013-1019.

20. Fan, Z.; Xiao, J.; Sun, K.; Chen, L.; Hu, Y.; Ouyang, J.; Ong, K. P.; Zeng, K.; Wang, J., Ferroelectricity of CH3NH3PbI3 Perovskite. J Phys Chem Lett 2015, 6 (7), 1155-61.

21. G, S.; Mahale, P.; Kore, B. P.; Mukherjee, S.; Pavan, M. S.; De, C.; Ghara, S.; Sundaresan, A.; Pandey, A.; Guru Row, T. N.; Sarma, D. D., Is CH3NH3PbI3 Polar? The Journal of Physical Chemistry Letters 2016, 7 (13), 2412-2419.

22. Sewvandi, G. A.; Kodera, K.; Ma, H.; Nakanishi, S.; Feng, Q., Antiferroelectric Nature of $\mathrm{CH} 3 \mathrm{NH} 3 \mathrm{PbI} 3-\mathrm{xClx}$ Perovskite and Its Implication for Charge Separation in Perovskite Solar Cells. Sci Rep 2016, 6, 30680. 
23. Yaffe, O.; Guo, Y.; Tan, L. Z.; Egger, D. A.; Hull, T.; Stoumpos, C. C.; Zheng, F.; Heinz, T. F.; Kronik, L.; Kanatzidis, M. G.; Owen, J. S.; Rappe, A. M.; Pimenta, M. A.; Brus, L. E., Local Polar Fluctuations in Lead Halide Perovskite Crystals. Physical Review Letters 2017, $118(13)$.

24. Zhao, X.; Park, N.-G., Stability Issues on Perovskite Solar Cells. Photonics 2015, 2 (4), 1139-1151.

25. Nagabhushana, G. P.; Shivaramaiah, R.; Navrotsky, A., Direct calorimetric verification of thermodynamic instability of lead halide hybrid perovskites. Proc Natl Acad Sci U S A 2016, 113 (28), 7717-21.

26. Babayigit, A.; Thanh, D. D.; Ethirajan, A.; Manca, J.; Muller, M.; Boyen, H. G.; Conings, B., Assessing the toxicity of $\mathrm{Pb}$ - and $\mathrm{Sn}$-based perovskite solar cells in model organism Danio rerio. Scientific Reports 2016, 6.

27. Babayigit, A.; Ethirajan, A.; Muller, M.; Conings, B., Toxicity of organometal halide perovskite solar cells. Nat Mater 2016, 15 (3), 247-51.

28. Li, Y.; Yang, K., High-throughput computational design of organic-inorganic hybrid halide semiconductors beyond perovskites for optoelectronics. Energy \& Environmental Science 2019, 12 (7), 2233-2243.

29. Filip, M. R.; Hillman, S.; Haghighirad, A. A.; Snaith, H. J.; Giustino, F., Band Gaps of the Lead-Free Halide Double Perovskites Cs2BiAgCl6 and Cs2BiAgBr6 from Theory and Experiment. J Phys Chem Lett 2016, 7 (13), 2579-85. 
30. Slavney, A. H.; Hu, T.; Lindenberg, A. M.; Karunadasa, H. I., A Bismuth-Halide Double Perovskite with Long Carrier Recombination Lifetime for Photovoltaic Applications. J Am Chem Soc 2016, 138 (7), 2138-41.

31. Volonakis, G.; Filip, M. R.; Haghighirad, A. A.; Sakai, N.; Wenger, B.; Snaith, H. J.; Giustino, F., Lead-Free Halide Double Perovskites via Heterovalent Substitution of Noble Metals. J Phys Chem Lett 2016, 7 (7), 1254-9.

32. McClure, E. T.; Ball, M. R.; Windl, W.; Woodward, P. M., Cs2AgBiX6(X=Br, Cl): New Visible Light Absorbing, Lead-Free Halide Perovskite Semiconductors. Chemistry of Materials 2016, $28(5), 1348-1354$.

33. Thind, A. S.; Kavadiya, S.; Kouhnavard, M.; Wheelus, R.; Cho, S. B.; Lin, L. Y.; Kacica, C.; Mulmudi, H. K.; Unocic, K. A.; Borisevich, A. Y.; Pilania, G.; Biswas, P.; Mishra, R., KBaTeBiO6: A Lead-Free, Inorganic Double-Perovskite Semiconductor for Photovoltaic Applications. Chemistry of Materials 2019, 31 (13), 4769-4778.

34. Huang, X.; Huang, S.; Biswas, P.; Mishra, R., Band Gap Insensitivity to Large Chemical Pressures in Ternary Bismuth Iodides for Photovoltaic Applications. Journal of Physical Chemistry C 2016, 120 (51), 28924-28932.

35. Xiao, Z.; Meng, W.; Wang, J.; Mitzi, D. B.; Yan, Y., Searching for promising new perovskite-based photovoltaic absorbers: the importance of electronic dimensionality. Mater. Horiz. 2017.

36. Walsh, A., Principles of Chemical Bonding and Band Gap Engineering in Hybrid OrganicInorganic Halide Perovskites. The Journal of Physical Chemistry C 2015, 119 (11), 5755-5760. 
37. Yin, W.-J.; Weng, B.; Ge, J.; Sun, Q.; Li, Z.; Yan, Y., Oxide perovskites, double perovskites and derivatives for electrocatalysis, photocatalysis, and photovoltaics. Energy \& Environmental Science 2019, 12 (2), 442-462.

38. Sun, W.; Dacek, S. T.; Ong, S. P.; Hautier, G.; Jain, A.; Richards, W. D.; Gamst, A. C.; Persson, K. A.; Ceder, G., The thermodynamic scale of inorganic crystalline metastability. Sci Adv 2016, 2 (11), e1600225.

39. Arca, E.; Lany, S.; Perkins, J. D.; Bartel, C.; Mangum, J.; Sun, W.; Holder, A.; Ceder, G.; Gorman, B.; Teeter, G.; Tumas, W.; Zakutayev, A., Redox-Mediated Stabilization in Zinc Molybdenum Nitrides. Journal of the American Chemical Society 2018, 140 (12), 4293-4301.

40. Van Aken, B. B.; Palstra, T. T. M.; Filippetti, A.; Spaldin, N. A., The origin of ferroelectricity in magnetoelectric YMnO3. Nature Materials 2004, 3 (3), 164-170.

41. Maeda, K.; Domen, K., New Non-Oxide Photocatalysts Designed for Overall Water Splitting under Visible Light. The Journal of Physical Chemistry C 2007, 111 (22), 7851-7861.

42. Fuertes, A., Chemistry and applications of oxynitride perovskites. Journal of Materials Chemistry 2012, 22 (8), 3293-3299.

43. Kim, Y.-I.; Woodward, P. M.; Baba-Kishi, K. Z.; Tai, C. W., Characterization of the Structural, Optical, and Dielectric Properties of Oxynitride Perovskites AMO2N (A = Ba, Sr, Ca; $\mathrm{M}=\mathrm{Ta}, \mathrm{Nb})$. Chemistry of Materials 2004, 16 (7), 1267-1276.

44. Wan, D.; Yan, B.; Chen, J.; Wu, S.; Hong, J.; Song, D.; Zhao, X.; Chi, X.; Zeng, S.; Huang, Z.; Li, C.; Han, K.; Zhou, W.; Cao, Y.; Rusydi, A.; Pennycook, S. J.; Yang, P.; 
Ariando; Xu, R.; Xu, Q.-H.; Wang, X. R.; Venkatesan, T., New Family of Plasmonic Photocatalysts without Noble Metals. Chemistry of Materials 2019, 31 (7), 2320-2327.

45. Wu, Y.; Lazic, P.; Hautier, G.; Persson, K.; Ceder, G., First principles high throughput screening of oxynitrides for water-splitting photocatalysts. Energy Environ. Sci. 2013, 6 (1), $157-$ 168.

46. Castelli, I. E.; Olsen, T.; Datta, S.; Landis, D. D.; Dahl, S.; Thygesen, K. S.; Jacobsen, K. W., Computational screening of perovskite metal oxides for optimal solar light capture. Energy Environ. Sci. 2012, 5 (2), 5814-5819.

47. Jain, A.; Ong, S. P.; Hautier, G.; Chen, W.; Richards, W. D.; Dacek, S.; Cholia, S.; Gunter, D.; Skinner, D.; Ceder, G.; Persson, K. A., Commentary: The Materials Project: A materials genome approach to accelerating materials innovation. APL Materials 2013, 1 (1), 011002.

48. Shannon, R. D., Revised Effective Ionic-Radii and Systematic Studies of Interatomic Distances in Halides and Chalcogenides. Acta Crystallographica Section A 1976, 32 (Sep1), 751767.

49. Fuertes, A., Prediction of Anion Distributions Using Pauling's Second Rule. Inorg. Chem. 2006, 45 (24), 9640-9642.

50. Morgan, P. E. D., Pauling's second crystal rule for nitrogen-substituted crystal structures. Journal of Materials Science 1986, 21 (12), 4305-4309.

51. Pauling, L., THE PRINCIPLES DETERMINING THE STRUCTURE OF COMPLEX IONIC CRYSTALS. J. Am. Chem. Soc. 1929, 51 (4), 1010-1026. 
52. Morgan, P. E. D.; Carroll, P. J.; Lange, F. F., Crystal structure of YSiO2N and a reappraisal of the "vaterite" type, YBO3. Materials Research Bulletin 1977, 12 (3), 251-259.

53. Shannon, R. D.; Prewitt, C. T., Synthesis and structure of phases in the In2O3 Ga2O3 system. Journal of Inorganic and Nuclear Chemistry 1968, 30 (6), 1389-1398.

54. Ouyang, L.; Yao, H.; Richey, S.; Xu, Y.-N.; Ching, W. Y., Crystal structure and properties of $\$\{\backslash \text { mathrm }\{\mathrm{YSiO}\}\}_{-}\{2\} \backslash$ mathrm $\{\mathrm{N}\} \$$. Physical Review B 2004, 69 (9), 094112.

55. Tohei, T.; Moriwake, H.; Murata, H.; Kuwabara, A.; Hashimoto, R.; Yamamoto, T.; Tanaka, I., Geometric ferroelectricity in rare-earth compounds $\$ \mathrm{R}\{\backslash \operatorname{text}\{\mathrm{GaO}\}\}_{-}\{3\} \$$ and \$R $\{\backslash \operatorname{text}\{\mathrm{InO}\}\}_{-}\{3\}$ \$. Physical Review B 2009, 79 (14), 144125.

56. Cohen, R. E., Origin of ferroelectricity in perovskite oxides. Nature 1992, 358 (6382), 136138.

57. Seshadri, R.; Hill, N. A., Visualizing the Role of Bi 6s "Lone Pairs" in the Off-Center Distortion in Ferromagnetic BiMnO3. Chemistry of Materials 2001, 13 (9), 2892-2899.

58. Attfield, J. P., Principles and Applications of Anion Order in Solid Oxynitrides. Crystal Growth \& Design 2013, 13 (10), 4623-4629.

59. Huang, Z. J.; Cao, Y.; Sun, Y. Y.; Xue, Y. Y.; Chu, C. W., Coupling between the ferroelectric and antiferromagnetic orders in $\$\{\backslash \text { mathrm }\{\text { YMnO }\}\}_{-}\{3\} \$$. Physical Review B 1997, $56(5), 2623-2626$.

60. Krukau, A. V.; Vydrov, O. A.; Izmaylov, A. F.; Scuseria, G. E., Influence of the exchange screening parameter on the performance of screened hybrid functionals. The Journal of Chemical Physics 2006, 125 (22), 224106. 
61. Leguy, A. M. A.; Azarhoosh, P.; Alonso, M. I.; Campoy-Quiles, M.; Weber, O. J.; Yao, J.; Bryant, D.; Weller, M. T.; Nelson, J.; Walsh, A.; van Schilfgaarde, M.; Barnes, P. R. F., Experimental and theoretical optical properties of methylammonium lead halide perovskites. Nanoscale 2016, 8 (12), 6317-6327.

62. He, J.; Franchini, C.; Rondinelli, J. M., Lithium Niobate-Type Oxides as Visible Light Photovoltaic Materials. Chemistry of Materials 2016, 28 (1), 25-29.

63. Mizoguchi, H.; Eng, H. W.; Woodward, P. M., Probing the Electronic Structures of Ternary Perovskite and Pyrochlore Oxides Containing Sn4+ or Sb5+. Inorganic Chemistry 2004, $43(5), 1667-1680$.

64. Pauling, L., THE NATURE OF THE CHEMICAL BOND. IV. THE ENERGY OF SINGLE BONDS AND THE RELATIVE ELECTRONEGATIVITY OF ATOMS. J. Am. Chem. Soc. 1932, 54 (9), 3570-3582.

65. Kotochigova, S., Levine, Z.H., Shirley, E.L., Stiles, M.D., and Clark, C.W., Atomic Reference Data for Electronic Structure Calculations (version 1.3). 2003.

66. Ganose, A. M. J., A. J.; Scanlon, D. O., sumo: Command-line tools for plotting and analysis of periodic ab initio calculations. The Journal of Open Source Software 2018, 28 (3), 717.

67. Wannier, G. H., The Structure of Electronic Excitation Levels in Insulating Crystals. Physical Review 1937, 52 (3), 191-197.

68. Baranowski, M.; Plochocka, P., Excitons in Metal-Halide Perovskites. Advanced Energy Materials n/a (n/a), 1903659. 
69. Glass, A. M.; von der Linde, D.; Negran, T. J., High-voltage bulk photovoltaic effect and the photorefractive process in LiNbO3. Applied Physics Letters 1974, 25 (4), 233-235.

70. Tan, L. Z.; Zheng, F.; Young, S. M.; Wang, F.; Liu, S.; Rappe, A. M., Shift current bulk photovoltaic effect in polar materials-hybrid and oxide perovskites and beyond. npj Computational Materials 2016, 2 (1).

71. Young, S. M.; Rappe, A. M., First principles calculation of the shift current photovoltaic effect in ferroelectrics. Phys Rev Lett 2012, 109 (11), 116601.

72. Young, S. M.; Zheng, F.; Rappe, A. M., First-principles calculation of the bulk photovoltaic effect in bismuth ferrite. Phys Rev Lett 2012, 109 (23), 236601.

73. Bhatnagar, A.; Roy Chaudhuri, A.; Heon Kim, Y.; Hesse, D.; Alexe, M., Role of domain walls in the abnormal photovoltaic effect in BiFeO3. Nature Communications 2013, 4 (1).

74. Tan, Z.; Hong, L.; Fan, Z.; Tian, J.; Zhang, L.; Jiang, Y.; Hou, Z.; Chen, D.; Qin, M.; Zeng, M.; Gao, J.; Lu, X.; Zhou, G.; Gao, X.; Liu, J.-M., Thinning ferroelectric films for highefficiency photovoltaics based on the Schottky barrier effect. NPG Asia Materials 2019, 11 (1), 20.

75. Machado, P.; Scigaj, M.; Gazquez, J.; Rueda, E.; Sanchez-Diaz, A.; Fina, I.; GibertRoca, M.; Puig, T.; Obradors, X.; Campoy-Quiles, M.; Coll, M., Band Gap Tuning of SolutionProcessed Ferroelectric Perovskite BiFe1-x Co x O3 Thin Films. Chem Mater 2019, 31 (3), 947954. 
76. Grinberg, I.; West, D. V.; Torres, M.; Gou, G.; Stein, D. M.; Wu, L.; Chen, G.; Gallo, E. M.; Akbashev, A. R.; Davies, P. K.; Spanier, J. E.; Rappe, A. M., Perovskite oxides for visiblelight-absorbing ferroelectric and photovoltaic materials. Nature 2013, 503 (7477), 509-512.

77. Nechache, R.; Harnagea, C.; Li, S.; Cardenas, L.; Huang, W.; Chakrabartty, J.; Rosei, F., Bandgap tuning of multiferroic oxide solar cells. Nat Photon 2015, 9 (1), 61-67.

78. Kresse, G.; Joubert, D., From ultrasoft pseudopotentials to the projector augmented-wave method. Physical Review B 1999, 59 (3), 1758-1775.

79. Kresse, G.; Furthmuller, J., Efficient iterative schemes for ab initio total-energy calculations using a plane-wave basis set. Physical Review B 1996, 54 (16), 11169-11186.

80. Perdew, J. P.; Burke, K.; Ernzerhof, M., Generalized Gradient Approximation Made Simple. Phys. Rev. Lett. 1996, 77 (18), 3865-3868.

81. Blöchl, P. E.; Jepsen, O.; Andersen, O. K., Improved tetrahedron method for Brillouinzone integrations. Physical Review B 1994, 49 (23), 16223-16233.

82. Mathew, K.; Montoya, J. H.; Faghaninia, A.; Dwarakanath, S.; Aykol, M.; Tang, H.; Chu, I.-h.; Smidt, T.; Bocklund, B.; Horton, M.; Dagdelen, J.; Wood, B.; Liu, Z.-K.; Neaton, J.; Ong, S. P.; Persson, K.; Jain, A., Atomate: A high-level interface to generate, execute, and analyze computational materials science workflows. Comp Mater Sci 2017, 139, 140-152.

83. Ong, S. P.; Richards, W. D.; Jain, A.; Hautier, G.; Kocher, M.; Cholia, S.; Gunter, D.; Chevrier, V. L.; Persson, K. A.; Ceder, G., Python Materials Genomics (pymatgen): A robust, open-source python library for materials analysis. Computational Materials Science 2013, 68 (Supplement C), 314-319. 
84. Baroni, S.; Resta, R., Ab initio calculation of the macroscopic dielectric constant in silicon. Physical Review B 1986, 33 (10), 7017-7021.

85. King-Smith, R. D.; Vanderbilt, D., Theory of polarization of crystalline solids. Phys. Rev. $B$ 1993, 47 (3), 1651-1654.

86. Campbell, B. J.; Stokes, H. T.; Tanner, D. E.; Hatch, D. M., ISODISPLACE: a web-based tool for exploring structural distortions. J. Appl. Crystallogr. 2006, 39 (4), 607-614.

87. Togo, A.; Tanaka, I., First principles phonon calculations in materials science. Scripta Mater. 2015, 108, 1-5.

88. Walsh, A.; Da Silva, J. L. F.; Yan, Y.; Al-Jassim, M. M.; Wei, S.-H., Origin of electronic and optical trends in ternary $\$\{\backslash \operatorname{text}\{\operatorname{In}\}\}_{-}\{2\}\{\backslash \operatorname{text}\{\mathrm{O}\}\}_{-}\{3\}\{(\mid \operatorname{text}\{\mathrm{ZnO}\})\}_{-}\{\mathrm{n}\} \$$ transparent conducting oxides $\$(n=1,3,5) \$$ : Hybrid density functional theory calculations. Physical Review $B$ 2009, $79(7), 073105$.

89. Filip, M. R.; Giustino, F., Computational Screening of Homovalent Lead Substitution in Organic-Inorganic Halide Perovskites. The Journal of Physical Chemistry C 2016, 120 (1), 166173.

90. Mi, Y.; Odaka, H.; Iwata, S., Electronic Structures and Optical Properties of ZnO, SnO2and In2O3. Japanese Journal of Applied Physics 1999, 38 (Part 1, No. 6A), 3453-3458. 